\title{
ALTERAÇÕES DE LINGUAGEM NAS FASES INICIAIS DA DOENÇA DE ALZHEIMER
}

\author{
Karin Zazo Ortiz', Paulo Henrique Ferreira Bertolucci
}

\begin{abstract}
RESUMO - A doença de Alzheimer (DA) está associada a alterações cognitivas, de linguagem e de comportamento que se agravam durante seu curso. $\mathrm{O}$ objetivo deste estudo foi verificar a presença de alterações de linguagem, em pacientes com DA em fase inicial. Foram avaliados 12 pacientes com diagnóstico de provável (DA). Todos tinham pontuação no Mini Exame do Estado Mental acima de 23 pontos. Os pacientes foram submetidos à aplicação do Teste de Boston e os resultados comparados aos da população normal. Todos os pacientes apresentaram alterações de linguagem. Foram encontradas diferenças estatisticamente significantes nas tarefas de Compreensão auditiva e na tarefa de denominação. Nas demais tarefas de expressão e compreensão oral, bem como nas de leitura e escrita, os pacientes tiveram desempenho similar aos normais. Embora com um grupo pequeno, esta investigação identificou alterações bem definidas de linguagem em uma fase bastante inicial da DA.
\end{abstract}

PALAVRAS-CHAVE: demência, doença de Alzheimer, linguagem.

\begin{abstract}
Language impairment in the early stages of Alzheimer's disease
ABSTRACT - Alzheimer disease (AD) is usually associated with cognitive, language and behavioral impairments, which can get more and more serious as the disease progresses. The aim of this study is to verify language disorders in the early stages of this disease. Twelve patients meeting criteria for problable AD were evaluated by the Boston test, and all of them scored more than 23 points on the Mini-Mental State Examination. Data acquired on this language evaluation were compared with the average of normal population data. All patients showed language disorders. Statistical differences were found in visual confrontating naming and auditory comphrension tasks. The patients performed well in writing and reading tasks. We believe that there might have had an interference in the patient's linguistic task performance due to their memory impairment. We could find language impairments in the early stages of AD.
\end{abstract}

KEY WORDS: dementia, Alzheimer's disease, language.

A doença de Alzheimer (DA) atinge no mínimo $5 \%$ da população de indivíduos com mais de 65 anos. Pacientes com DA apresentam alterações cognitivas, de linguagem e de comportamento que se agravam durante o curso da doença. As alterações de memória, no entanto, são as mais referidas pela família e as mais fortemente evidenciadas na avaliação clínica do paciente desde o início da doença. As alterações de fala e de linguagem, apesar de serem freqüentemente notadas, têm sido melhor estudadas nas duas últimas décadas ${ }^{1} \mathrm{e}$, da mesma forma, também têm sido melhor investigadas na prática clínica. $O$ uso da linguagem depende de conhecimentos múltiplos, incluindo sistemas de informação lingüística e sistemas de informação conceptual e perceptual não lingüísticas. Os conhecimentos são expressos através das estruturas fonológicas, sintáticas, semânticas e discursivas.

Por se tratar de um processo altamente complexo e elaborado, as alterações de linguagem podem ocorrer em qualquer um dos níveis descritos, e podem comprometer tanto a comunicação oral quanto a comunicação gráfica. Desta forma, a avaliação deve procurar identificar alterações nos aspectos emissivos e/ou compreensivos da linguagem em todas as suas vertentes. A maior parte dos estudos lingüísticos realizados com pacientes com $\mathrm{DA}$, buscou investigar, inicialmente, alterações no eixo léxico-semântico, principalmente alterações no acesso lexical ${ }^{2,3}$. A estes estudos iniciais, acrescentaram-se,

Departamento de Neurologia e Neurocirurgia da Universidade Federal de São Paulo - Escola Paulista de Medicina, São Paulo SP, Brasil (UNIFESP-EPM): 'Professor Adjunto Doutor da Disciplina de Distúrbios da Comunicação Humana do Departamento de Fonoaudiologia da UNIFESP-EPM; ${ }^{2}$ Professor Adjunto Doutor da Disciplina de Neurologia do Departamento de Neurologia e Neurocirurgia da UNIFESP-EPM.

Recebido 30 Junho 2004, recebido na forma final 29 Setembro 2004. Aceito 12 Novembro 2004.

Dr. Karin Zazo Ortiz - Rua Cunha 111/73 - 04037-030 São Paulo SP - Brasil. E-mail: Karin_zazo@hotmail.com 
paulatinamente, estudos descrevendo alterações semânticas ${ }^{4,5}$, sintáticas ${ }^{6}$ e discursivas ${ }^{7}$ tanto na comunicação oral quanto na comunicação gráfica ${ }^{8}$. Observamos também uma clara preocupação com a identificação precoce das possíveis alterações de linguagem ${ }^{1}$.

Nosso objetivo, neste estudo, foi verificar se poderíamos identificar alterações de linguagem precocemente, e quais seriam as manifestações mais comumente encontradas.

\section{MÉTODO}

O presente estudo foi realizado no Setor de Neurologia do Comportamento da Disciplina de Neurologia do Departamento de Neurologia e Neurocirurgia da Universidade Federal de São Paulo - Escola Paulista de Medicina. Contou com a participação livre e voluntária de 12 pacientes com DA, após assinatura de termo de consentimento Livre e Esclarecido. A pesquisa teve aprovação do Comitê de Ética em Pesquisa da Universidade Federal de São Paulo (parecer 1606/03).

Como o objetivo da pesquisa foi o de identificar alterações de linguagem que aparecem precocemente, nas fases iniciais da DA, todos os pacientes selecionados para este estudo, tinham pontuação no Mini Exame do Estado Mental (MEEM) acima de 23 pontos e $C D R=0,5$ ou 1,0. O tempo de evolução da doença variou de 2 a 6 anos, com média de 4 anos e 3 meses. Todos eram acompanhados no Setor de Neurologia do Comportamento e apresentavam diagnóstico de provável DA, de acordo com os critérios do DSM IV ${ }^{9}$ e NINCDS-ADRDA ${ }^{10}$, sendo excluídos os pacientes com transtornos psiquiátricos, vasculares ou pacientes com outros tipos de demência. Os pacientes foram então submetidos à avaliação de linguagem, através da aplicação do teste de Boston.

O teste de Boston, proposto por Goodglass e Kaplan ${ }^{11}$, é um teste de linguagem, composto por várias tarefas envolvendo a avaliação da compreensão e emissão, oral e gráfica. As tarefas realizadas e investigadas neste estudo são apresentadas a seguir:

Compreensão auditiva - Nesta etapa foram realizadas as seguintes tarefas: a) discriminação auditiva; b) identificação das partes do corpo; material ideacional complexo.

Expressão oral - Nesta etapa foram realizadas as seguintes tarefas: a) seqüências automatizadas; b) repetição de palavras; c) repetição de frases de alta freqüência; d) repetição de frases de baixa freqüência; e) leitura de palavras; f) denominação; g) denominação por confrontação visual

Compreensão da linguagem escrita - Nesta etapa foram realizadas as seguintes tarefas: a) discriminação de palavras e símbolos; b) associação fonética: b1) reconhe- cimento de palavras; b2) compreensão da soletração oral; c) emparelhamento palavras - figuras; d) leitura de parágrafos e sentenças.

Escrita - Nesta etapa foram realizadas as seguintes tarefas: a) reconhecimento de símbolos escritos: b1) escrita seriada; b2) ditado de $1^{\circ}$ nível; b) acesso lexical: b1) escrita: b2) escrita por confrontação visual; C: formulação escrita: sentenças escritas sob ditado.

Análise estatística - Para a verificação da distribuição da amostra em relação à curva de normalidade , utilizamos o teste de Kolmogorov - Smirnov. Para verificar as diferenças entre as médias dos escores obtidos com a população DA e os indivíduos normais, com distribuição paramétrica, utilizamos o teste $t$ de Student para uma amostra única. No caso de dados não-paramétricos, utilizamos o sign test. As médias da população sem DA utilizadas para a comparação neste estudo foram apresentadas por Radanovic e Mansur ${ }^{12}$. Para o teste Kolmogorov-Smirnov foi considerado valor estatisticamente significante $p<0,05$. Porém, para os testes de comparação entre as médias, devido à interferência da análise de comparações múltiplas, foi aplicada a correção de Bonferroni. A análise estatística foi realizada num computador pessoal, com o pacote estatístico SPSS para o windows (versão 11.5.1).

\section{RESULTADOS}

Os pacientes que participaram deste estudo tiveram pontuação no MEE superior a 23 pontos, com média de 25,5, - 1,3, limite inferior de 24 e superior de 28 pontos. As idades variaram entre 69 e 81 anos, com média de 74 anos. A escolaridade variou de primário completo a superior completo. Inicialmente, a amostra foi dividida em dois grupos: um com 6 pacientes com primário completo e um com 6 pacientes com curso superior completo. A análise intergrupos que comparou os dados obtidos neste estudo, com as médias publicadas para indivíduos normais considerando-se a escolaridade ${ }^{1}$, não mostrou diferenças significantes. Deste modo, não consideramos a divisão dos grupos de acordo com a escolaridade, e todas as análises apresentadas a seguir, se referem ao grupo de 12 pacientes.

Na Tabela 1, apresentamos a análise descritiva dos resultados obtidos na nossa amostra no teste de Boston, bem como as médias publicadas para indivíduos normais por Radanovic e Mansur ${ }^{1}$.

A comparação do desempenho dos pacientes com DA leve e sem DA nas tarefas do teste de Boston são apresentadas nas Tabelas 2 e 3 . A diferença entre a média dos escores na tarefa de compreensão auditiva-material ideacional complexo e a mé- 
Tabela 1. Performance do grupo de indivíduos sem DA e com DA leve no teste de Boston.

\begin{tabular}{|c|c|c|c|c|c|c|}
\hline & Média & $\begin{array}{l}\text { Desvio } \\
\text { Padrão }\end{array}$ & Mediana & $\begin{array}{l}\text { Limite } \\
\text { Inferior }\end{array}$ & $\begin{array}{l}\text { Limite } \\
\text { Superior }\end{array}$ & $\begin{array}{c}\text { População } \\
\text { normal } \\
\text { (Radanovic } \\
\text { \& Mansur, 2002) }\end{array}$ \\
\hline \multicolumn{7}{|l|}{ Compreensão auditiva } \\
\hline Discriminação auditiva & 69,0 & 4,2 & 71 & 59 & 72 & 70,4 \\
\hline Identificação das partes do corpo & 17,9 & 1,5 & 18 & 15 & 20 & 19,2 \\
\hline Material ideacional complexo & 7,5 & 1,7 & 8 & 5 & 10 & 10,9 \\
\hline \multicolumn{7}{|l|}{ Expressão oral } \\
\hline Seqüências automatizadas & 7,3 & 0,5 & 7 & 7 & 8 & 7,8 \\
\hline Repetição de palavras & 9,7 & 0,6 & 10 & 8 & 10 & 9,9 \\
\hline Repetição de frases - alta freqüência & 7,2 & 1,0 & 7 & 5 & 8 & 7,8 \\
\hline Repetição de frases - baixa freqüência & 7,1 & 0,8 & 7 & 6 & 8 & 7,8 \\
\hline Leitura de palavras & 29,6 & 1,2 & 30 & 26 & 30 & 29,2 \\
\hline Denominação & 27,0 & 0,0 & 27 & 27 & 27 & 26,7 \\
\hline Denominação por confrontação visual & 96,9 & 8,6 & 96 & 79 & 110 & 112,0 \\
\hline Leitura oral de sentenças & 9,8 & 0,4 & 10 & 9 & 10 & 9,8 \\
\hline \multicolumn{7}{|l|}{ Compreensão da linguagem escrita } \\
\hline Associação fonética. reconhecimento de palavras & 8,0 & 0,0 & 8 & 8 & 8 & 7,9 \\
\hline Associação fonética-soletração & 4,6 & 2,2 & 5 & 2 & 8 & 6,7 \\
\hline Emparelhamento palavra-figura & 9,6 & 0,5 & 10 & 9 & 10 & 9,7 \\
\hline Leitura de palavras e sentenças & 9,2 & 0,9 & 9 & 8 & 10 & 9,5 \\
\hline \multicolumn{7}{|l|}{ Escrita } \\
\hline Reconhec. símbolos escritos-escrita seriada & 43,4 & 3,3 & 45 & 35 & 46 & 44,0 \\
\hline Reconhec. símbolos escritos-ditado $1^{\circ}$ nível & 14,7 & 0,5 & 15 & 14 & 15 & 14,5 \\
\hline Acesso léxicol-escrita & 8,9 & 1,0 & 9 & 7 & 10 & 9,4 \\
\hline Acesso léxicol-denominação por confrontação & 9,6 & 0,8 & 10 & 8 & 10 & 9,8 \\
\hline Formulação escrita- sentenças sob ditado & 10,7 & 1,7 & 11 & 6 & 12 & 11,7 \\
\hline
\end{tabular}

dia dos escores referidos para a população normal ${ }^{1}$ foram estatisticamente significantes para o nível de $0,02 \%$, e assim, pudemos inferir que o escore médio dos pacientes com DA leve foi realmente inferior aos da população normal obtidos por Radanovic e Mansur ${ }^{1}(7,5 \pm 1,7$ para o grupo DA leve e $10,9 \pm 1,4$ para o grupo controle, $t(10)=-6,6, p$ $<0,001,95 \% \mathrm{Cl}-4,5 \mathrm{a}-2,2)$ apresentados na Tabela 2. Além disso, os valores da média da tarefa de denominação por confrontação visual também foram estatisticamente inferiores no grupo DA $(96,9-$ 8,6 , versus $112,0-4,7, t(10)=-5,8, p<0,001,95 \% \mathrm{Cl}$ - 20,9 para - 9,3).

\section{DISCUSSÃO}

Todos os pacientes apresentaram alterações de linguagem.

Nossos achados devem ser examinados, no entanto, com algumas limitações. Inicialmente, o nú- mero restrito de indivíduos com DA avaliados neste estudo, impossibilitou a verificação de diferenças significantes entre o desempenho dos dois grupos na maior parte das tarefas realizadas. Por outro lado, pudemos identificar claramente um desempenho inferior do grupo DA nas tarefas de compreensão do material ideacional complexo e de denominação por confrontação visual.

A tarefa de compreensão auditiva, material ideacional complexo, consiste de 12 itens com 2 perguntas cada, totalizando 24 questões. Os primeiros 4 itens são relativos à compreensão de frases, sendo os demais, compostos por perguntas relativas à compreensão de 3 textos. Assim, analisando-se o conjunto de itens da tarefa, pôde-se verificar que os pacientes tiveram falhas na compreensão de sentenças complexas e, sobretudo, de textos. Em relação à dificuldade que pacientes com DA possam apresentar em relação à compreensão de sentenças encontramos, na literatura compul- 
Tabela 2. Comparação do desempenho dos pacientes com DA leve e sem DA nas tarefas do teste de Boston: discri minação auditiva, identificação das partes do corpo, compreensão do material ideacional complexo, repetição de frases, denominação por confrontação visual, associação fonética e acesso lexico-escrita.

\begin{tabular}{|c|c|c|c|c|c|c|}
\hline \multirow{2}{*}{$\begin{array}{l}\text { Total } \\
\text { Discriminação auditiva }\end{array}$} & \multirow{2}{*}{$\begin{array}{c}\mathrm{t} \\
-\end{array}$} & \multirow{2}{*}{$\begin{array}{c}\text { df } \\
10,00\end{array}$} & \multirow{2}{*}{$\begin{array}{c}p \\
0,312\end{array}$} & \multirow{2}{*}{$\begin{array}{c}\begin{array}{c}\text { Diferença } \\
\text { entre as } \\
\text { médias }\end{array} \\
-1,355\end{array}$} & \multicolumn{2}{|c|}{$\begin{array}{l}95 \% \text { Intervalo } \\
\text { de confiança } \\
\text { da diferença }\end{array}$} \\
\hline & & & & & $-4,187$ & 1,478 \\
\hline & 1,066 & 0 & & & & \\
\hline \multirow[t]{2}{*}{ Identificação das partes do corpo } & - & 10,00 & 0,012 & $-1,336$ & $-2,311$ & $-0,362$ \\
\hline & 3,055 & 0 & & & & \\
\hline \multirow[t]{2}{*}{ Material ideacional complexo } & - & 10,00 & $<0,001$ & $-3,4$ & $-4,493$ & $-2,216$ \\
\hline & 6,564 & 0 & & & & \\
\hline \multirow[t]{2}{*}{ Repetição de frases - alta freqüência } & - & 10,00 & 0,063 & $-0,618$ & $-1,278$ & 0,041 \\
\hline & 2,089 & 0 & & & & \\
\hline \multirow[t]{2}{*}{ Repetição de frases- baixa freqüência } & - & 10,00 & 0,018 & $-0,709$ & $-1,268$ & $-0,151$ \\
\hline & 2,829 & 0 & & & & \\
\hline \multirow[t]{2}{*}{ Denominação por confrontação visual } & - & 10,00 & $<0,001$ & $-15,091$ & $-20,858$ & $-9,324$ \\
\hline & 5,830 & 0 & & & & \\
\hline \multirow[t]{2}{*}{ Associação fonética-soletração } & - & 10,00 & 0,010 & $-2,064$ & $-3,513$ & $-0,614$ \\
\hline & 3,172 & 0 & & & & \\
\hline \multirow[t]{2}{*}{ Acesso léxico-escrita } & - & 10,00 & 0,150 & $-0,491$ & $-1,193$ & 0,211 \\
\hline & 1,559 & 0 & & & & \\
\hline
\end{tabular}

$p<0.0023$ indica significância estatística

Tabela 3. Comparação do desempenho dos pacientes com DA leve e sem DA nas tarefas do teste de Boston: reconhecimento dos símbolos escritos- escrita seriada e ditado, seqüências automatizadas, acesso lexical gráfico, emparelhamento palavra-figura, repetição de palavras, leitura de palavras e sentenças, leitura de palavras e leitura oral de sentenças.

\begin{tabular}{lcc}
\hline Total & $\begin{array}{c}\text { Proporção } \\
\text { observada }\end{array}$ & $\mathrm{p}$ \\
\hline Reconhecimento de símbolos escritos-escrita seriada & 0,45 & 1,000 \\
Reconhecimento de símbolos escritos-ditado 1 $^{\circ}$ nível & 0,27 & 0,227 \\
Seqüências automatizadas & 0,73 & 0,227 \\
Acesso lexical gráfico- confrontação visual & 0,18 & 0,065 \\
Emparelhamento palavra-figura & 0,36 & 0,549 \\
Formulação escrita- sentenças sob ditado & 0,64 & 0,549 \\
Repetição de palavras & 0,18 & 0,065 \\
Leitura de palavras e sentenças & 0,55 & 1,000 \\
Leitura de palavras & 0,09 & 0,012 \\
leitura oral de sentenças & 0,18 & 0,065
\end{tabular}

Proporção do teste $p \leq 0,5$ 
sada, diversos estudos abordando esta temática. De fato, nos últimos anos, tem havido um interesse importante na relação entre memória e a compreensão de sentenças ${ }^{13,14}$. A idéia de que a alça fonológica teria um papel importante na compreensão da linguagem tem sido aceita por grande número de pesquisadores. Uma das primeiras explicações sobre o papel da alça fonológica na compreensão de sentenças foi a de que algumas sentenças dependeriam inicialmente de uma análise detalhada e complexa da estrutura sintáxica para que se identificasse o papel dos substantivos nas mesmas. Este seria, por exemplo, o processamento das sentenças na voz passiva que podem ser revertidas em ativas ${ }^{15}$.

Há ainda alguns autores que acreditam que o componente semântico ${ }^{13}$ ou lingüístico-semânti$\mathrm{CO}^{16,17}$ também possa interferir na compreensão de sentenças. As sentenças que constam do material ideacional complexo no teste de Boston, são sentenças que efetivamente merecem uma análise detalhada do conteúdo sintático em relação com o processamento semântico. Por exemplo: "um quilo de farinha é mais pesado do que 2 ?", merece uma análise inicial da estrutura da sentença, reversão da voz ativa em passiva e reflexão sobre o conteúdo, para uma resposta correta. Assim, acreditamos ser provável que a memória de trabalho e a memória semântica possam ter interferido neste processamento, apesar de, neste caso, devido à simplicidade do conteúdo semântico, a memória de trabalho possa ter interferido mais do que uma alteração no processamento semântico de entrada. De fato, há consenso em se considerar que tipos de sentenças diferentes podem ter processamentos também distintos ${ }^{13,18}$. No processamento de sentenças, as informações sintáticas e semânticas são extraídas de cada uma das palavras como são percebidas e a estrutura sintática e a interpretação são construídas baseando-se em cada uma das palavras dentro de uma extensão de frase possível ${ }^{19}$. No entanto, para alguns tipos de sentenças, é possível um atraso neste processamento, devido a dificuldade na construção sintática ou no vocabulário. Nestas situações, se realizássemos uma análise baseando-se no modelo de memória, especificamente pensando-se na memória de trabalho, a informação deve ser estocada na alça fonológica até que o executivo central possa processá-la.

Nos pacientes com DA, encontramos alterações da memória, considerando-se seus subsistemas, precocemente. Desta forma, tal alteração parece interferir em determinados processamentos de lin- guagem, como a alteração na compreensão de sentenças identificada neste estudo. Assim, nossos achados, uma vez que avaliamos especificamente pacientes com DA em fase inicial, parecem sugerir que a alteração de memória interfere no processamento lingüístico e, conseqüentemente, na compreensão de sentenças. De fato, tais pacientes apresentaram desempenho alterado em testes específicos de memória (dados não apresentados neste estudo).

Da mesma forma, a dificuldade que pacientes com DA encontraram na compreensão de textos, corrobora achados de estudos que abordaram compreensão textual em pacientes com $\mathrm{DA}^{20}$. De fato, as dificuldades na compreensão discursiva destes pacientes parecem também relacionadas a outras habilidades cognitivas, incluindo dificuldades com a compreensão de pressupostos, subentendidos e inferências, além de ausência da preditividade na compreensão de sentenças ambíguas. Além disso, mesmo quando pacientes com DA conseguem estocar e recuperar as informações de um dado discurso, pode-se perceber que eles são capazes de lembrar melhor de informações essenciais do que de detalhes. Há ampla discussão sobre as dificuldades de compreensão de sentenças e de discursos em pacientes com DA. A literatura na área vem demonstrando que embora possam ser similares, os processamentos de sentenças e de discursos não parecem compartilhar dos mesmos recursos $\operatorname{cognitivos}^{21,22}$. Além disso, parece que o processamento do discurso está mais marcadamente afetado nestes pacientes, sendo mais relacionado, neste caso, às falhas da memória de trabalho. Apesar das falhas na memória de trabalho, como discutido anteriormente, poderem interferir no processamento de sentenças, é mais clara a participação desta memória na compreensão de textos do que na compreensão de sentenças.

Em relação à alteração encontrada na nomeação por confrontação visual, sabemos que é possível identificar precocemente falhas de nomeação em pacientes com $D^{1}{ }^{1}$, sendo este achado comum na prática clínica. Pesquisas com pacientes com DA têm demonstrado déficits no processamento léxico-semântico, e nestes casos têm-se discutido se esta alteração estaria mais relacionada a perda da informação semântica ou à dificuldades no acesso léxico. De fato, é bem reconhecido que falhas de nomeação podem ter múltiplas causas. Elas podem estar relacionadas a falhas no sistema semântico, sistema léxico-semântico e/ou léxico-fonológico ${ }^{23-25}$. Po- 
rém, nos casos de nomeação por confrontação visual, deve-se também considerar a hipótese da alteração de nomeação existir por falha no processo de reconhecimento visual da figura ou uma falha no acesso ao sistema semântico decorrente do processamento visual ${ }^{26-29}$. Em relação à controvérsia relativa a este tópico, há uma tendência em se considerar tal prejuízo relativo à perda da informação semântica ${ }^{1,4,3}$. Apesar desta tendência, nos pacientes avaliados neste estudo, não parece ter sido a perda da informação semântica o fator causal da diferença estatisticamente significante encontrada no desempenho entre o grupo DA em fase inicial e os controles. Como pudemos observar, os pacientes com DA avaliados neste estudo tiveram desempenho geral nas diversas tarefas lingüísticas tão bom quanto os indivíduos normais, inclusive se olharmos mais especificamente a tarefa de denominação por acesso semântico. Neste tipo de tarefa, os pacientes tinham que ouvir uma pergunta e acessar a palavra correspondente ao saber semântico, por exemplo: onde nós vemos as horas? De que cor é a grama? O desempenho adequado dos pacientes nesta tarefa, sugere, ao menos, que não há alteração de entrada entre a informação auditiva, estando adequado o acesso ao semântico e deste para o léxico-fonológico de saída. Desta forma, na denominação por confrontação visual, poderíamos ter ainda duas hipóteses para justificar o desempenho do grupo com DA: falha no processo de reconhecimento visual da figura ou ainda na passagem da informação do sistema semântico ao léxico-fonológico de saída ou no próprio sistema léxico-fonológico. Porém, ainda observando-se o desempenho dos pacientes nas demais tarefas do teste, verificamos que não houve alteração estatisticamente significante entre os pacientes DA e o grupo-controle nas tarefas de denominação escrita, sugerindo que o reconhecimento visual não poderia justificar a falha observada na denominação oral. Assim, nossos dados sugerem dificuldade na busca lexicol ou léxico-fonológica.

Sabemos que pacientes com DA apresentam, precocemente queixas relativas à memória. Muitos estudos sugeriram a participação da memória de trabalho, especialmente a alça fonológica e do executivo central em diversos outros aspectos no processamento da linguagem como produção da fala, aquisição de vocabulário e leitura ${ }^{24,30}$. Da mesma forma, sabemos que o bom desempenho lingüístico depende da preservação de funções cognitivas como memória e atenção.
Apesar das tarefas lingüísticas mais alteradas identificadas neste estudo provavelmente dependerem, como vimos, dos subsistemas de memória, sobretudo da memória de trabalho/alça fonológica e da chamada memória semântica ou buffer semântico, para serem adequadamente realizadas, a avaliação de linguagem permitiu a verificação precoce de alterações do processamento lingüístico já na fase inicial da doença.

Este é um estudo-piloto, pois analisamos um número restrito de casos. É possível que possamos identificar outras alterações de linguagem, não identificadas neste estudo, precocemente, quando tivermos avaliado número maior de pacientes com DA em fase inicial. As alterações de linguagem comumente evidenciadas em pacientes com DA, freqüentemente afetam a atividade comunicativa gerando, na maior parte dos pacientes, o isolamento e aumentando o risco de institucionalização precoce $^{31}$. Esta é a razão para a importância da identificação precoce de alterações lingüísticas.

Em conclusão, neste estudo, pudemos identificar alterações de linguagem em pacientes com DA em fase inicial. Acreditamos que a avaliação de linguagem realizada precocemente possa corroborar para a compreensão das habilidades lingüístico-cognitivas alteradas nestes pacientes.

\section{REFERÊNCIAS}

1. Giles E, Patterson K, Hodges JR. Performance on the Boston Cookie Theft picture description task in patients with early dementia of the Alzheimer's type: missing information. Aphasiology 1996;10:395-498.

2. Bayles KA. Language function in senile dementia. Brain Language 1982;16:265-280

3. Hodges JR, Salmon DP, Butters N. The nature of naming deficits in Alzheimer's and Huntingdon's disease. Brain 1991;114:1547-1558.

4. Chertkhon H, Bub D. Semantic memory loss in Alzheimer's type: what do various measures measure? Brain 1990;397-417.

5. Martin A, Fedio P. Word production and comprehension in Alzheimer's disease: the breakdown of semantic knowledge. Brain Language 1983;19:124-141.

6. Appell J, Kertesz A, Fisman M. A study of language functioning in Alzheimer patients. Brain Language 1982;17:73-91.

7. Obler L, Albert M, Helm-Estabrooks N. Empty speech in Alzheimer's disease and fluent aphasia. J Speech Hearing Res 1985;28:405-410.

8. LuzzattiC, Laiacona M, Agazzi D. Multiple patterns of writing disorders in dementia of the Alzheimer type and their evolution. Neuropsychologia 2003;41:759-772.

9. DSM-IV. Manual Diagnóstico e Estatístico de Transtornos Mentais. Trad. Dayse Batista, 4a ed. Porto Alegre: Artes Médicas, 1995.

10. Mckhann G, Drachman D, Folstein M,Katzman R, Price D, Stadlan E M. Clinical diagnosis of Alzheimer's disease: report of the NINCDSADRDA Work Group under the auspices of Department of Health and Human Services Task Force on Alzheimer's Disease. Neurology 1984;34,939-944

11. Goodglass H, Kaplan E. The assessment of aphasia and related disorders. Philadelphia: Lea \& Fegiber, 1972.

12. Radanovic M, Mansur LL. Performance of a Brazilian population sample in the Boston diagnostic aphasia examination: a pilot study. Braz J Med Biol Res 2002;35:305-317. 
13. Martin RC, Romani C. Verbal working memory and sentence comprehension: a multiple-components view. Neuropsychology 1994;8:506-523.

14. Collette F, Van Der Linden M, Poncelet M. Working memory, long term memory and language processing: issues and future directions. Brain Language 2000;71:46-51.

15. Caramazza A, Basili AG, Koller J, Berndt RS. An investigation of repetition and language processing in a case of conduction aphasia. Brain Language 1981;14:235-271.

16. Berndt RS, Mitchum CC, Wayland S. Patterns of sentence comprehension in aphasia: a consideration of three hypotheses. Brain Language 1997;60:197-221.

17. Grossman M, White-Devine T. Sentence comprehension in Alzheimer's disease. Brain Language 1998;62:186-201.

18. Pulvermüller F. Syntatic circuits: how does the brain create serial order in sentences? Brain Language 2000;71:194-199.

19. Waters GS, Caplan D. The capacity theory of sentence comprehension: critique of just and carpenter. Psychol Rev 1996;103:761-772.

20. Orange JB, Kertesz A. Discourse analyses and dementia. Brain Language 2000;71:172-174.

21. Almor A, Kempler D, MacDonald M, Andersen E, Tyler L. Why do Alzheimer patients have difficulty with pronouns? Working memory, semantics, and reference in comprehension and production in Alzheimer's disease. Brain Language 1999;67:202-227.

22. MacDonald M, Almor A, Henderson V, Kempler D, Andersen E. Assessing working memory and language comprehension in Alzheimer's disease. Brain Language 2001;78:17-42.
23. Hillis AE, Rapp BC, Caramazza A. When a rose is a rose in speech but a tulip in writing. Cortex 1999;35:337-356.

24. Martin RC, Breedin SD, Damian MF. The relation of phoneme discrimination, lexical access, and short-term memory: a case study and interactive activation account. Brain Language 1999;437-482.

25. Miceli G, Amitrano A, Capasso R, Caramazza A. The treatment of anomia resulting from output lexical damage: analysis of two cases. Brain Language 1996;52:150-174.

26. Chanoine V, Teixeira-Ferreira C, Demonet JF, Nespoulous JL, Poncet M. Optic aphasia with pure alexia: a mild form of visual associative agnosia? A case study. Cortex 1998;34:437-448.

27. Coslett HB, Saffran EM. Optic aphasia and the right hemisphere: a replication and extinction. Brain Language 1992;43:148-161.

28. Iorio L, Falanga A, Fragassi NA, Grossi D. Visual associative agnosia and optic aphasia: a single case study and review of the syndromes. Cortex 1992;28:23-37.

29. Lhermitte E, Beauvois MF. A visual-speech disconnexion syndrome: report of a case with optic aphasia, agnosic alexia and colour agnosia. Brain 1973;96:695-714.

30. Martin N, Saffran EM. Language and auditory-verbal short-term memory impairments: evidence for common underlying processes. Cogn Neuropsychol 1997;14:641-682.

31. Small JA, Gutman G. Recommended and reported use of communication strategies in Alzheimer caregiving. Alzh Dis Associat Disord 2002;16:270278. 